\title{
Engaging Patients with Depression in Treatment Continuity
}

\author{
Dan J. Stein
}

Received: September 15, 2021 / Accepted: December 17, 2021 / Published online: March 5, 2022

(C) The Author(s) 2022

\section{ABSTRACT}

When conceptualising engagement with treatment, it may be useful to contrast 'disease', which refers to underlying psychobiological dysfunction, with 'illness', which describes the experience of the person with that disease. Knowledge of disease, as well as understanding of illness, including patients' explanatory models of symptoms, may be useful in facilitating shared decision-making. Ideally, physicians are able to integrate evidence-based medicine with values-based medicine by combining the best research evidence with patients' unique needs and preferences. This, in turn, requires taking a systematic approach to the assessment of a range of domains (i.e. symptom profile, clinical subtype, severity and comorbidity) in individuals with depression, and individualising treatment accordingly. While data are now available from a range of randomised clinical trials addressing treatments for depressive symptoms, it is also notable that a decrease in symptom severity does not necessarily correlate with an increase in functioning, which highlights the need to monitor patients

D. J. Stein $(\bowtie)$

SAMRC Unit on Risk \& Resilience, Department of Psychiatry \& Neuroscience Institute, Groote Schuur Hospital, University of Cape Town, Anzio Road, Observatory, 7925 Cape Town, South Africa e-mail: dan.stein@uct.ac.za for the effect of treatment on a range of outcomes including comorbid anxiety, emotional responsiveness, and sleep quality. Importantly, recent epidemiological data emphasise the importance of persistence with treatment; most patients with major depression who persist with treatment eventually feel helped.

Keywords: Depression; Shared decisionmaking; Treatment outcomes; Treatment persistence; Patient engagement

\section{Key Summary Points}

Patient engagement with treatment is facilitated by shared decision-making which combines the best research evidence with the patient's unique values.

Individualised treatment of depression requires systematic assessment of a range of domains; findings may be used to tailor interventions appropriately.

The majority of adults with a lifetime history of treated depression eventually receive treatment that they consider helpful, but many stop seeking treatment after early unhelpful treatment; persistence is therefore key. 


\section{INTRODUCTION}

The majority of adults with a lifetime history of treated depression eventually obtain a treatment that they consider helpful, but many stop seeking treatment after early unhelpful treatment [1]. This paper begins by providing a conceptual framework for thinking about how to engage the patient with depression in treatment, arguing that shared decision-making is key. It then discusses a range of domains that should be assessed in the patient with depression, in order to individualise treatment, and so optimise outcomes. Finally, the paper examines some of the evidence from pharmacotherapy and psychotherapy trials of depression and concludes by emphasizing the importance of persistence with treatment.

\section{CONCEPTUAL FRAMEWORK}

Arthur Kleinman, a psychiatrist and anthropologist, has highlighted the distinction between 'disease' and 'illness'; disease refers to psychobiological mechanisms, whereas illness refers to the experience of the person with disease [2]. Illness is shaped by the familial, social, and cultural environment the person lives in, with attendant perceptions, values, and explanations of that disease. Similarly, since the time of William Osler, it has been emphasised that physicians need to understand not only the disease but also the person with the condition. This point was further highlighted by the World Psychiatric Association's development of a patient-centred initiative, in which "the whole person of the patient in [their] context" is at the centre of clinical care [3].

These considerations are relevant to the framework of shared decision-making, i.e. a way of thinking and doing things in which the people using health and social services are seen as equal partners in planning, developing, and monitoring care to make sure it meets their needs. The essential elements of shared decisionmaking include defining the problem, presenting options, discussing the pros and cons and the patient's preferences and abilities, clarifying their understanding, and then making or explicitly deferring a management decision [4]. A related framework emphasises the value of integrating evidence-based medicine with values-based medicine, i.e. combining the best research evidence with the patient's unique values, including their needs and preferences [5].

Indeed, psychiatry increasingly aspires to personalised medicine, in which treatment is tailored to the individual based on their genes, environment, and sociocultural context [6]. In day-to-day clinical practice, person-centred care and shared decision-making are facilitated by the physician taking time to ask patients for their own explanation of why they are experiencing their symptoms and, given that explanatory model, what they consider to be the best way forward. Therefore, key early questions in the clinical interview include: "What do you think is going on?" and "Based on that, what do you think is the best way forward?". The Diagnostic and Statistical Manual of Mental Disorders (DSM)-5 cultural formulation interview may also be useful for ensuring a holistic understanding of the illness [7].

\section{DIAGNOSTIC CONSIDERATIONS}

Recent editions of the DSM have advanced the field by providing a widely used set of diagnostic criteria for depression. Nevertheless, the presentation of depression is very heterogeneous, and there are multiple ways in which patients can meet DSM-5 criteria. Current treatment guidelines may result in only partial treatment success in part because they are not sufficiently individualised.

In order to tailor treatment for depression effectively, physicians need to take a rigorous and systematic approach to the assessment of a range of domains [8]. These include symptom profile, clinical subtypes, severity, neurocognition, functioning and quality of life, clinical staging, personality traits, antecedent and concomitant psychiatric conditions such as anxiety, physical comorbidities, family history, early and recent environmental exposures, protective factors and resilience, and dysfunctional cognitive schemas [8]. While findings from 
translational neuroscience may ultimately be useful for individualising the treatment of depression, currently domains such as symptom profile, clinical subtypes, and severity and comorbidity are more relevant to case conceptualisation and choice of treatment. Individualised treatment based on these domains may facilitate patient engagement and improve treatment outcomes.

\section{PHARMACOTHERAPY CONSIDERATIONS}

Meta-analyses have been useful in synthesizing the current evidence base on pharmacotherapy for depression. A large network meta-analysis of 21 different antidepressant drugs found that, in head-to-head comparisons, some agents (agomelatine, amitriptyline, escitalopram, mirtazapine, paroxetine, venlafaxine, and vortioxetine) were more effective than others, with odds ratios ranging from 1.19 to 1.96 (Fig. 1) [9]. The same analysis found that some antidepressants (agomelatine, citalopram, escitalopram, fluoxetine, sertraline, and vortioxetine) were more acceptable than others, based on the proportion of patients who discontinued treatment for any reason [9], with odds ratios in this analysis ranging from 0.43 to 0.77 .

Notably, patients with depression can experience a spectrum of outcomes from response to remission to recovery, and a decrease in symptom severity does not necessarily correlate with an increase in function [10, 11]. In addition, antidepressant agents with a comparable effect on depression symptoms may not have the same effects on features of depression that are important to patients; these include emotional responsiveness, cognitive function, and sleep quality. For example, in a randomised, doubleblind comparison of agomelatine and escitalopram, the two agents had a similar effect on symptoms of depression, but patients receiving agomelatine experienced less emotional blunting and felt better on waking compared with patients receiving escitalopram [12].

Anxious depression is associated with greater severity, increased suicidality, and worse outcomes than depression that is not associated with anxiety [13], so robust treatment of patients with depression and comorbid anxiety is needed. Overall, there are limited data suggesting that one class of antidepressant agent is more effective than another for anxious depression [14, 15]. However, a meta-analysis of data from six randomised controlled trials of agomelatine found a significantly greater early effect (weeks 2-4) on anxiety severity in patients with depression receiving agomelatine compared with patients receiving placebo, selective serotonin reuptake inhibitors, or serotonin norepinephrine reuptake inhibitors [16]. The difference in the impact of agomelatine on the Hamilton Depression Rating Scale subscores compared with placebo was significant in the overall study population and in patients with more anxiety at baseline (Fig. 2), but in depressed patients with more severe anxiety, agomelatine had a significantly greater effect on anxiety and depressive symptoms than comparator antidepressants [16].

\section{PSYCHOTHERAPY CONSIDERATIONS}

Meta-analyses have also been useful in synthesising the current evidence base on psychotherapy for depression. Many patients with depression will benefit from a combined approach to treatment that incorporates both psychotherapy and pharmacotherapy. A network meta-analysis of 101 studies in 11,910 patients showed that combined treatment was more effective in achieving response $(\geq 50 \%$ reduction in depression score) at the end of treatment compared with either psychotherapy alone [relative risk [RR] of response 1.27; 95\% confidence intervals (CI) 1.14-1.39] or pharmacotherapy alone (RR 1.25; 95\% CI 1.14-1.37) [17].

An exciting development in psychotherapy has been the adaptation of psychotherapeutic strategies developed in high-income countries for use in low- and middle-income countries [18]. The recent Lancet Commission on Global Mental Health and Sustainable Development emphasises the importance of mental health for sustainable development around the world [19]. The Lancet Commission highlighted a number 
OR $(95 \% \mathrm{Crl})$

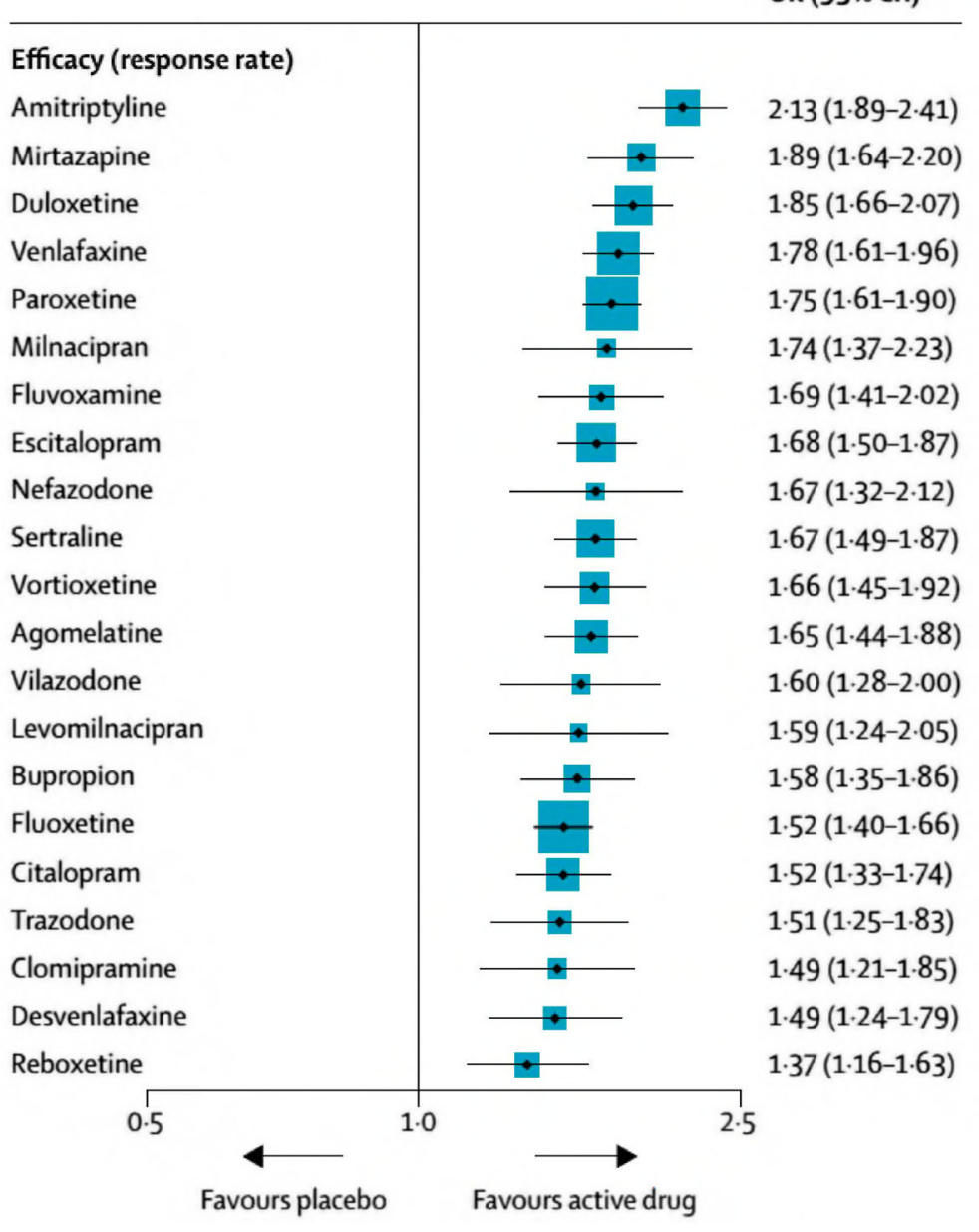

Fig. 1 Forest plot of a large network meta-analysis. Antidepressant agents were compared with placebo [9]. OR odds ratio, CrI credible interval. Reprinted from Cipriani et al. [9]

of key points, including the need for a spectrum approach to conceptualising mental illness, the cost-efficacy of interventions for common mental disorders, and the importance of a human rights approach to mental health services [20].

\section{THE IMPORTANCE OF PERSISTENCE}

Interesting data have emerged from the World Mental Health Surveys about what patients with depression perceive as helpful in the treatment of their depression [1]. A total of $68.2 \%$ $(n=2726)$ of adults with a lifetime history of treated major depression obtained treatment that they considered helpful; others stopped seeking treatment after early unhelpful treatment. Most patients (93.9\%) were helped if they persisted through 10 treatment professionals, but only $21.5 \%$ of patients showed this level of persistence. While randomised controlled trials provide important data on treatment efficacy, these epidemiological data on treatment helpfulness emphasise the crucial importance of treatment persistence [1].

\section{CONCLUSION}

The treatment of depression requires an individualised approach to both assessment and treatment. Clinicians need to evaluate not only symptoms of depression but also a range of 
HAM-D subscore (10+11)

Agomelatine vs placebo -Total population

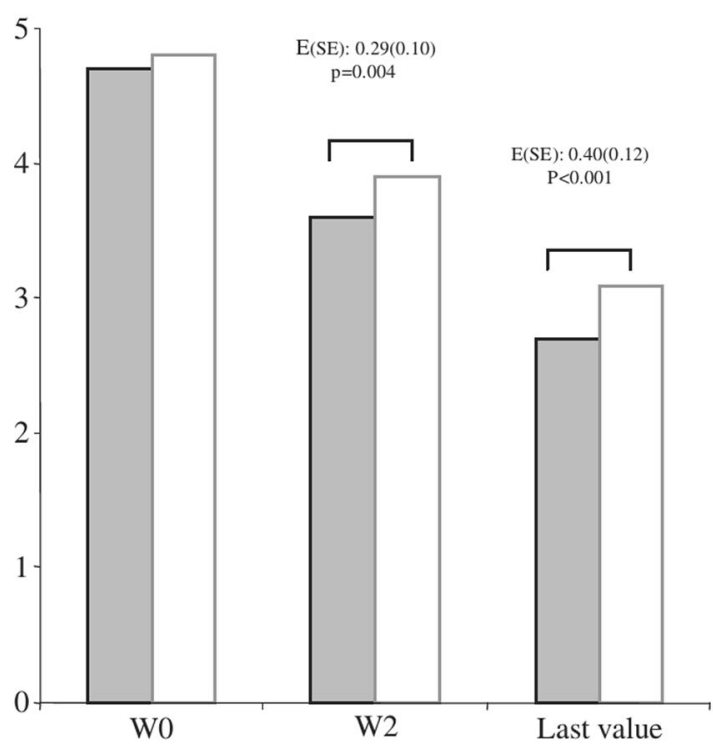

$\square$ agomelatine $25-50 \mathrm{mg}(\mathrm{n}=358)$

Placebo $(n=363)$

Fig. 2 Effect of agomelatine on anxiety-related subscores (questions 10 and 11) of the Hamilton Depression Rating Scale in a pooled analysis of three randomised, doubleblind, placebo-controlled studies [16]. E(SE) Effect size

considerations that impact the patient's life, including comorbid conditions and cognitive and functional impairments. Meta-analyses suggest that some antidepressants are more efficacious or better tolerated, and that there may be differences between agents on key outcomes such as anxiety, sleep, and cognition. Patients may need to seek help from a number of different health professionals before they find a treatment for depression that helps them; treatment persistence should therefore be encouraged.

\section{ACKNOWLEDGEMENTS}

Funding. This article is based on a presentation given by Prof. Stein at a satellite symposium entitled Road to Recovery for Depressed

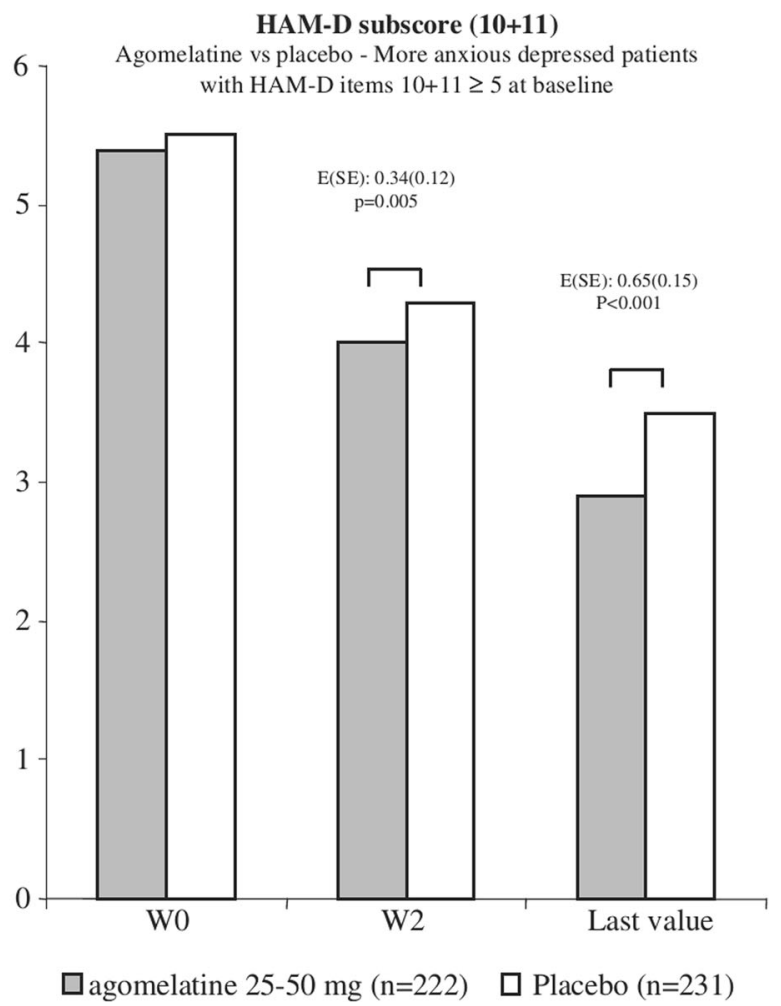

(standard error), $W$ week. Used with permission of John Wiley \& Sons Ltd, from Ref. [16]; permission conveyed through Copyright Clearance Center, Inc

Patients: From Treatment Initiation to Continuity. The symposium formed part of the programme of the WPA World Congress of Psychiatry, held 10-13 March 2021. The satellite symposium was sponsored by Servier, which also provided funding for medical writing assistance. This supplement has been sponsored by Servier.

Medical Writing. The author would like to thank Catherine Rees of Springer Healthcare Communications, who wrote the first draft of the manuscript based on the corresponding presentation at WPA World Congress of Psychiatry 2021. Funding for the writing was provided by Servier.

Authorship. The named author of this article meets the International Committee of Medical Journal Editors (ICMJE) criteria for authorship for this article, takes responsibility 
for the integrity of the work as a whole and has given their approval for this version to be published.

Author Contributions. Prof. Stein prepared the content of the presentation on which this article was based and reviewed and revised all drafts of the manuscript.

Prior Presentation. This article is based on a presentation given by the author at a satellite symposium entitled Road to Recovery for Depressed Patients: From Treatment Initiation to Continuity. The symposium formed part of the programme of the WPA World Congress of Psychiatry, held virtually from 10-13 March 2021. The content within the presentation and article is based on work that has been previously published.

Disclosures. Prof. Stein has received research grants and/or consultancy honoraria from Johnson \& Johnson, Lundbeck, Servier, Takeda and Vistagen. Servier has participated in the development of this article.

Compliance with Ethics Guidelines. This article is based on previously conducted studies and does not contain any new studies with human participants or animals performed by any of the authors.

Open Access. This article is licensed under a Creative Commons Attribution-NonCommercial 4.0 International License, which permits any non-commercial use, sharing, adaptation, distribution and reproduction in any medium or format, as long as you give appropriate credit to the original author(s) and the source, provide a link to the Creative Commons licence, and indicate if changes were made. The images or other third party material in this article are included in the article's Creative Commons licence, unless indicated otherwise in a credit line to the material. If material is not included in the article's Creative Commons licence and your intended use is not permitted by statutory regulation or exceeds the permitted use, you will need to obtain permission directly from the copyright holder. To view a copy of this licence, visit http://creativecommons.org/licenses/by$\mathrm{nc} / 4.0 /$.

\section{REFERENCES}

1. Harris MG, Kazdin AE, Chiu WT, Sampson NA, Aguilar-Gaxiola S, Al-Hamzawi A, et al. Findings from world mental health surveys of the perceived helpfulness of treatment for patients with major depressive disorder. JAMA Psychiat. 2020;77(8): 830-41.

2. Kleinman A, Eisenberg L, Good B. Culture, illness, and care: clinical lessons from anthropologic and cross-cultural research. Ann Intern Med. 1978;88(2):251-8.

3. Mezzich JE. World Psychiatric Association perspectives on person-centered psychiatry and medicine. Int J Integr Care. 2010;10(Suppl):e003.

4. Makoul G, Clayman ML. An integrative model of shared decision making in medical encounters. Patient Educ Couns. 2006;60(3):301-12.

5. Fulford KW. The value of evidence and evidence of values: bringing together values-based and evidence-based practice in policy and service development in mental health. J Eval Clin Pract. 2011;17(5):976-87.

6. Stein D. Problems of living. Perspectives from philosophy, psychiatry, and cognitive-affective science. 1st ed. Waltham, MA: Academic Press; 2021.

7. Lewis-Fernandez R, Krishan Aggarwal N, Ladson H, Kirmayer JL. DSM-5 ${ }^{\circledR}$ handbook on the cultural formulation interview. Washington, DC: American Psychiatric Association Pub; 2015.

8. Maj M, Stein DJ, Parker G, Zimmerman M, Fava GA, De Hert $\mathrm{M}$, et al. The clinical characterization of the adult patient with depression aimed at personalization of management. World Psychiatry. 2020;19(3):269-93.

9. Cipriani A, Furukawa TA, Salanti G, Chaimani A, Atkinson LZ, Ogawa Y, et al. Comparative efficacy and acceptability of 21 antidepressant drugs for the acute treatment of adults with major depressive disorder: a systematic review and network metaanalysis. Lancet. 2018;391(10128):1357-66.

10. Lee Y, Rosenblat JD, Lee J, Carmona NE, Subramaniapillai $M$, Shekotikhina $M$, et al. Efficacy of antidepressants on measures of workplace functioning in major depressive disorder: a systematic review. J Affect Disord. 2018;227:406-15. 
11. McKnight PE, Kashdan TB. The importance of functional impairment to mental health outcomes: a case for reassessing our goals in depression treatment research. Clin Psychol Rev. 2009;29(3): 243-59.

12. Corruble E, de Bodinat C, Belaidi C, Goodwin GM, Agomelatine Study G. Efficacy of agomelatine and escitalopram on depression, subjective sleep and emotional experiences in patients with major depressive disorder: a 24-wk randomized, controlled, double-blind trial. Int J Neuropsychopharmacol. 2013;16(10):2219-34.

13. Rao S, Zisook S. Anxious depression: clinical features and treatment. Curr Psychiatry Rep. 2009;11(6):429-36.

14. Ionescu DF, Niciu MJ, Richards EM, Zarate CA Jr. Pharmacologic treatment of dimensional anxious depression: a review. Prim Care Companion CNS Disord. 2014;16(3):13r01621.

15. Papakostas GI, Stahl SM, Krishen A, Seifert CA, Tucker VL, Goodale EP, et al. Efficacy of bupropion and the selective serotonin reuptake inhibitors in the treatment of major depressive disorder with high levels of anxiety (anxious depression): a pooled analysis of 10 studies. J Clin Psychiatry. 2008;69(8):1287-92.

16. Stein DJ, Picarel-Blanchot F, Kennedy SH. Efficacy of the novel antidepressant agomelatine for anxiety symptoms in major depression. Hum Psychopharmacol. 2013;28(2):151-9.

17. Cuijpers $\mathrm{P}$, Noma $\mathrm{H}$, Karyotaki E, Vinkers $\mathrm{CH}$, Cipriani A, Furukawa TA. A network meta-analysis of the effects of psychotherapies, pharmacotherapies and their combination in the treatment of adult depression. World Psychiatry. 2020;19(1): 92-107.

18. Stein DJ, Bass JK, Hoffmann SG, editors. Global mental health and psychotherapy: adapting psychotherapy for low- and middle-income countries. 1st ed. London: Academic; 2019.

19. Patel V, Saxena S, Lund C, Thornicroft G, Baingana $\mathrm{F}$, Bolton P, et al. The Lancet Commission on global mental health and sustainable development. Lancet. 2018;392(10157):1553-98.

20. Stein D, Singh I. Global mental health and neuroethics. 1st ed. London: Elsevier; 2020. p. 310. 